\title{
As consequências do desemprego para os rendimentos de reemprego: uma análise para diferentes condições do mercado de trabalho
}

\author{
Mauricio Cortez Reis ${ }^{1}$
}

\section{Resumo}

Este artigo tem como objetivo analisar as consequências de transições do emprego para o desemprego sobre os rendimentos dos trabalhadores no Brasil, comparando os resultados em diferentes contextos do mercado de trabalho. As evidências encontradas usando dados longitudinais da PNAD contínua para o período entre 2012 e 2017 mostram que as transições do emprego para o desemprego nos períodos em que a situação do mercado de trabalho é mais favorável parecem relacionadas com reduções pequenas e em muitos casos não significativas nos rendimentos dos que conseguem se reempregar no intervalo de um ano. O mesmo tipo de transição nos períodos em que a situação do mercado de trabalho é desfavorável, porém, parece ter como consequência perdas nos rendimentos entre $10 \%$ e 15\%, de acordo com as estimativas. Uma parte dessas perdas se deve ao fluxo mais intenso para empregos informais que geralmente ocorre nesses períodos.

\section{Palavras-Chave}

Desemprego. Rendimentos do trabalho. Condições do mercado de trabalho.

\begin{abstract}
This paper aims to investigate labor earnings losses of workers who become unemployed in Brazil, comparing the results for different stages of the business cycle. Estimates using longitudinal data from the continuous PNAD for the period between 2012 and 2017 show that workers who transited from employment to unemployment and were reemployed during a period of strong labor market conditions experienced small and usually non-significant earnings declines. However, earnings losses are estimated between 10 and 15\% for those who became unemployed and were able to find a new job under weak labor market conditions. Part of this reduction seems to be due to the workers flow to jobs in the informal sector, which is higher during periods of high unemployment rates compared to times of better labor market conditions.
\end{abstract}

1 Pesquisador - Instituto de Pesquisa Econômica Aplicada End.: Av. Presidente Antônio Carlos, 51(1409) - Rio de Janeiro/RJ - Brasil - CEP: 20020-010 E-mail: mauricio.reis@ipea.gov.br - ORCID: https://orcid.org/0000-0002-5551-0249. Recebido: 19/02/2020. Aceito: 07/08/2020

Editor responsável: Fabio Waltenberg

(c) (i) (\$) Esta obra está licenciada com uma Licença Creative Commons Atribuição-Não Comercial 4.0 Internacional. 


\section{Keywords}

Unemployment. Labor earnings. Labor Market conditions.

\section{JEL Classification}

J30. J63. E24.

\section{Introdução}

Entre o primeiro trimestre de 2015 e o primeiro trimestre de 2017, a taxa de desemprego no Brasil passou de $6,7 \%$ para $11,6 \%$. Essa diferença de 5 pontos percentuais representa, nesse último período, cerca de 4 milhões de desempregados. ${ }^{1}$ Embora o fluxo do emprego para o desemprego seja apenas um dos componentes do aumento recente da taxa de desemprego no Brasil, essas evidências reforçam a preocupação com as consequências que a perda do emprego pode ter para o trabalhador, em particular com uma conjuntura desfavorável no mercado de trabalho. Além da perda dos rendimentos do trabalho, com implicações diretas para o orçamento familiar, a literatura econômica também destaca que o processo até encontrar um novo emprego pode ser demorado, e que mesmo para os que conseguem se reempregar, os rendimentos do trabalho são normalmente inferiores em comparação com os que recebiam anteriormente. ${ }^{2}$

O objetivo deste artigo é analisar como saídas do emprego para o desemprego influenciam os rendimentos de reemprego no Brasil. Essa análise procura se concentrar na comparação desse efeito entre diferentes períodos do ciclo econômico, explorando as intensas oscilações observadas nos últimos anos. Mais especificamente, pretende-se investigar em que medida as perdas nos rendimentos para os que transitam para o desemprego nos períodos em que o mercado de trabalho está mais favorável diferem daquelas que ocorrem em períodos de taxa de desemprego mais elevada. $\mathrm{Na}$ próxima seção, são apresentados alguns estudos da escassa literatura que analisa as consequências da saída do emprego para os rendimentos de reemprego no Brasil. Não é do conhecimento do autor, no entanto, que essa questão tenha sido investigada para diferentes condições do mercado de trabalho no Brasil.

1 Valores calculados para a PNAD contínua considerando indivíduos com idade entre 21 e 60 anos. A Figura A.l no apêndice mostra as taxas de desemprego entre o primeiro trimestre de 2012 e o terceiro trimestre de 2017 no Brasil.

2 Ver, por exemplo, Farber $(2015,2017)$. 
Na literatura econômica, podem ser encontradas várias evidências mostrando que trabalhadores que se tornam desempregados, quando voltam a se empregar, costumam experimentar reduções nos seus rendimentos. ${ }^{3}$ Diversos fatores podem estar por trás desse resultado. Uma razão que costuma ser muito enfatizada é baseada no fato das transições do emprego para o desemprego estarem associadas a uma perda, pelo menos parcial, do capital humano que o trabalhador acumulou durante o período que passou empregado. É possível também que os trabalhadores, ao se tornarem desempregados, reduzam os seus salários de reserva, que podem ser representados como uma função dos custos de busca, de outras rendas não advindas do próprio trabalho, e da expectativa por novas ofertas de trabalho, e passem a aceitar empregos de pior qualidade, levando a reduções nos rendimentos. Outro elemento que costuma ser mencionado é baseado na hipótese de que o desemprego pode ser interpretado pelos empregadores como um sinal negativo da capacidade produtiva do trabalhador. ${ }^{4}$

A análise empírica neste artigo procura se beneficiar dos dados longitudinais da PNAD contínua (Pesquisa Nacional por Amostra de Domicílios contínua), que permitem acompanhar os indivíduos por um período de 5 entrevistas em 5 trimestres consecutivos. Com isso, para os indivíduos que participam de todas as entrevistas, é possível obter a situação no mercado de trabalho em cada um desses períodos. Separando os indivíduos que transitaram do emprego para o desemprego e posteriormente se reempregaram daqueles que permaneceram empregados durante as cinco entrevistas, são estimados modelos baseados nos métodos de diferença-em-diferenças e de propensity score matching com diferença-em-diferenças. As estimações são realizadas com observações referentes a diferentes períodos de tempo dentro do intervalo entre 2012 e 2017, o que permite comparações entre os efeitos estimados sobre os rendimentos das transições dos trabalhadores para o desemprego em diferentes situações do mercado de trabalho. Entre 2012 e 2014, a taxa de desemprego oscilou entre 5 e 7\%, mas a partir do primeiro trimestre de 2015 foi iniciada uma trajetória de aumento bastante acentuada. Três anos depois, a taxa de desemprego chegou próxima de 12\%, como mostra a Figura A.l.

De acordo com os resultados, transições para o desemprego durante períodos mais favoráveis do mercado de trabalho estão associadas a redu-

3 Jacobson et al. (1993), Ruhm (1991) e Couch e Placzek (2010), entre vários outros.

4 Kletzer (1998) e Manzoni and Mooi-Reci (2020) descrevem as abordagens teóricas normalmente utilizadas para explicar o impacto do desemprego sobre os rendimentos de reemprego. 
ções de magnitude pequena nos rendimentos, que em alguns casos nem chegam a apresentar significância estatística. Já nos períodos em que a situação do mercado de trabalho é pior, as reduções nos rendimentos são maiores, representando uma queda estimada entre $10 \%$ e $15 \%$ na maioria dos casos. Esse comportamento é semelhante ao observado na literatura internacional que investiga as consequências da perda do emprego sobre os rendimentos em diferentes estágios do ciclo econômico. Parte dessas reduções estimadas nos rendimentos para o Brasil pode ser atribuída ao fluxo para empregos no setor informal, que se torna mais intenso para os trabalhadores reempregados durante os períodos de desemprego elevado. Trabalhadores com carteira que se reempregam nessa mesma situação têm, em média, reduções nos rendimentos, mas não tão intensas quanto as experimentadas pelos com carteira que se reempregam como trabalhadores por conta própria ou empregados sem carteira. Essa mesma tendência de aumento nas transições para empregos com remunerações mais baixas em relação a empregos melhor remunerados é observada por Schmieder $e t$ al. (2020) para a Alemanha, e por Farber $(2015,2017)$ para a recessão de 2007-09 nos Estados Unidos.

O restante do artigo está organizado da seguinte maneira. Na seção 2 são apresentadas algumas evidências da literatura econômica para o efeito do desemprego sobre os rendimentos de reemprego. Na seção 3 é descrita a construção da amostra com base nos dados da PNAD contínua, e na seção 4 são apresentados os métodos de estimação usados na análise empírica. Em seguida, na seção 5, são mostrados os resultados estimados, enquanto a seção 6 contém as principais conclusões do artigo.

\section{Algumas evidências na literatura econômica sobre variações nos rendimentos como consequência do desemprego}

Existe uma literatura bastante vasta dedicada a estimar as perdas nos rendimentos decorrentes de transições para o desemprego, com evidências empíricas para diversos países. As magnitudes dessas perdas tendem a variar bastante entre os estudos. Nesta seção são mostrados alguns resultados para essa relação de maneira geral e para diferentes estágios do ciclo econômico. 
Jacobson et al. (1993) estimam uma redução de 40\% nos salários como resultado do desemprego nos Estados Unidos. Já Topel (1990) estima uma redução de 17\%, enquanto Ruhm (1991) e Stevens (1997) encontram quedas entre 14 e $18 \%$ e em torno de 30\%, respectivamente, também para os Estados Unidos. Todos esses resultados se referem ao reemprego no prazo de um ano. Para períodos mais longos os efeitos são menores, mas persistem. Couch e Placzek (2010) encontram uma queda de aproximadamente 1/3 dos salários para o reemprego após um ano, e entre 13 e $15 \%$ para o reemprego após o período de 6 anos.

Os resultados obtidos para países europeus geralmente mostram efeitos menores que os encontrados para os Estados Unidos, de acordo com Burda e Mertens (2001). Esses autores estimam, para a Alemanha, reduções de $3,6 \%$ nos rendimentos um ano após o desemprego. Gregory e Jukes (2001) encontram rendimentos $10 \%$ menores em relação aos recebidos anteriormente para os trabalhadores reempregados no Reino Unido, sendo que esse diferencial tende a desaparecer depois de dois anos. Hijzen et al. (2010), entretanto, também para trabalhadores no Reino Unido, obtêm quedas bem mais expressivas, oscilando entre 14 e 35\% após um período de 5 anos. Os efeitos também podem ser bastante persistentes. Verho (2020) analisa os impactos da perda do emprego durante a recessão econômica na Finlândia durante o início da década de noventa. Acompanhando os trabalhadores por um período de 16 anos, o autor mostra que, mesmo depois de mais de uma década, os rendimentos para os homens ainda são entre 5 e $8 \%$ menores do que no período anterior à crise.

Para os países em desenvolvimento, também podem ser encontradas algumas evidências empíricas. Amarante et al. (2014) apresentam resultados indicando uma redução de $14 \%$ nos rendimentos dos trabalhadores no Uruguai como consequência desse efeito. ${ }^{5}$ Resultados para o Brasil usando informações da PME (Pesquisa Mensal de Emprego) para as seis principais regiões metropolitanas do país durante o período entre 1982 e 2002 também apontam para uma queda nos rendimentos (Hoek 2006). Nesse estudo, os trabalhadores da indústria que entram no desemprego experimentam uma queda de 50\% nos rendimentos de reemprego em relação ao que recebiam um ano antes, quando ainda estavam empregados. Para os indivíduos com idade entre 35 e 40 anos, as reduções nos rendimentos

${ }^{5}$ Amarante et al. (2014) usam uma amostra de trabalhadores formais do setor privado a partir de dados administrativos dos registros do sistema de seguridade social uruguaio. 
chegam a 70\%. Menezes-Filho (2003), com dados administrativos da RAIS (Relação Anual de Informações Sociais) para os trabalhadores formais brasileiros com longa duração no emprego, também encontra uma redução de 50\% nos rendimentos um ano após o ingresso no desemprego. Para trabalhadores com até 3 anos no emprego essa queda é estimada em 10\%.

Diversos trabalhos procuram investigar também como as reduções nos rendimentos resultantes da passagem pelo desemprego variam ao longo dos ciclos econômicos. As evidências, em geral, indicam perdas bem mais acentuadas nos períodos de recessão. Para os Estados Unidos, Davis e Wachter (2011) mostram que nos períodos de expansão da economia os rendimentos de reemprego são $23 \%$ menores um ano após a saída do emprego, enquanto nos períodos de recessão os rendimentos diminuem $40 \%$. Farber (2015), também com dados para os Estados Unidos, estima uma redução de $12 \%$ para os trabalhadores em tempo integral que se reempregaram igualmente com jornada em tempo integral, durante o período 20072009, marcado por uma intensa recessão. Embora essa não seja considerada pelo autor como uma queda desproporcional para os padrões históricos, quando comparada a outros períodos recessivos, a redução estimada para o período 2011-13, posterior a recessão, já é bem menor, 3,6\%. Farber (2015) destaca, porém, que um efeito importante da recessão nos Estados Unidos foi o de aumentar a participação relativa dos empregos em tempo parcial no fluxo de reemprego.

Efeitos cíclicos também são encontrados por Schmieder et al. (2020) para a Alemanha, com perdas nas recessões correspondendo ao dobro das que são registradas nos períodos de expansão. Esses autores também mostram que uma parcela importante das reduções nos rendimentos ocorre pela saída de empregos de melhor qualidade e reemprego em postos de pior qualidade, sendo esse processo mais intenso durante as recessões. Resultados estimados para a Finlândia por Korkeamäki and Kyyrä (2014) mostram que a perda mediana nos rendimentos durante o período recessivo de 1992 foi de 15\%, enquanto no período de recuperação em 1997 a perda de emprego não implicou queda nos rendimentos. Amarante et al. (2014) mostram que nos períodos de recessão as reduções nos rendimentos dos trabalhadores reempregados no Uruguai são estimadas em $34 \%$, bem mais intensas, portanto, que as reduções verificadas de maneira geral, que se situam em torno de $15 \%$. 


\section{Os dados}

$\mathrm{Na}$ análise empírica, são usados dados da PNAD contínua (Pesquisa Nacional por Amostra de Domicílios contínua), calculada pelo IBGE (Instituto Brasileiro de Geografia e Estatística), para o período compreendido entre o primeiro trimestre de 2012 e o terceiro trimestre de 2017. Em novembro de 2017, entrou em vigor uma ampla reforma da legislação trabalhista. Para evitar a possível influência dessas mudanças nos movimentos dos trabalhadores entre as situações de emprego e desemprego, os dados se restringem até o terceiro trimestre de 2017.

Na PNAD contínua, os domicílios são entrevistados por cinco trimestres consecutivos, sendo possível, dessa forma, seguir o mesmo indivíduo durante esse período. São selecionados para esse estudo apenas os indivíduos ocupados e com rendimentos do trabalho positivos durante a primeira entrevista da PNAD contínua. A amostra também é restrita aos indivíduos ocupados e com rendimentos do trabalho positivos no período da quinta entrevista, que é realizada um ano após a primeira. Além disso, apenas os indivíduos com informações sobre a situação no mercado de trabalho para as cinco entrevistas na PNAD contínua são incluídos na análise, que também é limitada àqueles com idade entre 21 e 60 anos no início da sua participação na pesquisa.

Os indivíduos na amostra são divididos em dois grupos, definidos da seguinte maneira. O grupo de tratamento contém os indivíduos que se encontravam desempregados em pelo menos uma das entrevistas 2, 3 e 4, enquanto o grupo de controle é formado pelos indivíduos que se encontravam ocupados em todas essas três entrevistas da PNAD contínua. Pela periodicidade da PNAD contínua, somente são observadas diretamente as transições entre os trimestres. Dessa maneira, é possível que indivíduos no grupo de controle tenham passado pelo desemprego, desde que tenham transitado do emprego para o desemprego, e posteriormente retornado ao emprego, tudo isso dentro do intervalo de apenas um trimestre.

A PNAD contínua não oferece informação sobre o motivo para o indivíduo ter saído do emprego. As características produtivas dos indivíduos podem estar associadas a diferenças no motivo, como saída voluntária ou demissão por justa causa, por exemplo. Como a definição do grupo de tratamento exige que o indivíduo se encontre desempregado, ou seja, não esteja ocupado e se encontre na busca por um emprego, a probabilidade de 
ano no emprego durante a primeira entrevista da PNAD contínua entre os indivíduos do grupo de tratamento, e aos rendimentos que, nesse mesmo período, ou seja, antes da entrada do grupo de tratamento no desemprego, são 50\% maiores para trabalhadores no grupo de controle.

\section{Método}

A primeira parte da análise empírica, baseada em Farber (1993), consiste em aplicar o método de diferença-em-diferenças. Para isso, são comparadas as variações no log dos rendimentos da primeira para a quinta entrevista na PNAD contínua entre os trabalhadores nos grupos de tratamento e controle. Mais especificamente, pretende-se estimar a seguinte equação:

$$
\Delta \ln \left(\text { rend }_{i}\right)=\beta_{o}+\beta_{1} D_{i}+\gamma X_{i}+u_{i}
$$

Onde $\Delta \ln \left(\right.$ rend $\left._{i}\right)=\ln \left(\right.$ rend $\left._{i t+4}\right)-\ln \left(\right.$ rend $\left._{i t}\right)$, sendo rend $d_{i t}$ e rend $d_{i t+4}$ os rendimentos no trabalho principal do indivíduo $i$ no período $t$ e quatro trimestres depois, e $D_{i}$ é uma variável dummy igual a um para os indivíduos que experimentaram pelo menos um trimestre de desemprego entre a primeira e a quinta entrevista, e igual a zero para os que permaneceram empregados durante as cinco entrevistas.

$\mathrm{O}$ vetor $\mathrm{X}_{\mathrm{i}}$ representa algumas características do indivíduo i, como idade, idade ao quadrado, gênero, escolaridade, região de residência, e duração no emprego anterior. Essas variáveis contidas em $\mathrm{X}_{\mathrm{i}}$ usam o período da primeira entrevista como referência. A Equação (1) é estimada separadamente para cada um dos anos de entrada do indivíduo na amostra entre 2012 e 2016.

O segundo método de estimação usado é baseado no escore de propensão. Nesse caso, cada indivíduo no grupo de tratamento, que passou por um período de desemprego, é comparado com indivíduos no grupo de controle, que permaneceram empregados, com características similares as suas. Como Rosenbaum e Rubin (1983) mostram, essa associação entre os indivíduos dos dois grupos pode se basear na probabilidade de se tornar desempregado em função das suas características observadas, $\operatorname{Prob}(D=1 \mid Z)$. 
Dessa forma, o estimador de escore de propensão pode ser definido como:

$$
m=\frac{1}{N_{1}} \sum_{i \in I_{1} \cap S_{P}}\left[Y_{1 i}-\hat{E}\left(Y_{0 i} \mid T_{i}=1, \operatorname{Pr}\left(T=1 \mid Z_{i}\right)\right)\right]
$$

onde: $Y_{1 i}$ representa os rendimentos de um indivíduo i no grupo de tratamento, e $Y_{0 j}$ representa os rendimentos para um indivíduo j no grupo de controle. Além disso, $\mathrm{I}_{1}$ é o conjunto de indivíduos no grupo de tratamento, $\mathrm{I}_{0}$ é o conjunto de indivíduos no grupo de controle, $\mathrm{S}_{\mathrm{p}}$ é a região de suporte comum, que consiste nas observações cujos escores de propensão pertencem a interseção dos suportes para os grupos de tratamento e controle, e $\mathrm{N}_{1}$ é o número de indivíduos no conjunto $I_{1} \cap S_{P}$.

Para estimar $m$ na Equação (2), considera-se que $\hat{E}\left(Y_{0 i} \mid T_{i}=1, \operatorname{Pr}\left(T=1 \mid Z_{i}\right)\right)=$ $\sum_{j \in I_{0} \cap S_{P}} w(i, j) Y_{0 j}$, ou seja, o rendimento de cada indivíduo no conjunto $I_{1} \cap S_{P}$ é associado ao valor de uma média ponderada entre indivíduos no grupo de controle, em que os pesos dependem da distância entre $P_{i}=\operatorname{Pr}\left(D=1 \mid Z_{i}\right)$ e $P_{j}=\operatorname{Pr}\left(D=1 \mid Z_{j}\right)$.

O estimador na Equação (2) supõe que a seleção dos trabalhadores que passaram por um período de desemprego é determinada pelas características observadas contidas em Z. Entretanto, fatores não observados podem ser diferentes para os indivíduos nos grupos de tratamento e controle, e provocarem viés no estimador. O estimador de diferença-em-diferenças com matching proposto por Heckman et al. (1997) permite controlar para as características não observadas dos indivíduos em cada um dos dois grupos que são constantes ao longo do tempo.

Supondo que para cada trabalhador no grupo de tratamento exista pelo menos um análogo no grupo de controle, dadas as características em $Z,{ }^{7} \mathrm{e}$ que $E\left(Y_{0 t}-Y_{0 t^{\prime}} \mid Z, D=1\right)=E\left(Y_{0 t}-Y_{0 t^{\prime}} \mid Z, D=0\right)$, onde t e t' representam os períodos correspondentes a primeira e a quinta entrevista, o estimador de diferença-em-diferenças com matching é:

$d d m=\frac{1}{N_{1}} \sum_{i \in I_{1} \cap S_{P}}\left[\left(Y_{1 t i}-Y_{1 t^{\prime} i}\right)-\sum_{j \in I_{0} \cap S_{P}} w(i, j)\left(Y_{0 t j}-Y_{0 t^{\prime} j}\right)\right]$

Onde os pesos w(i,j) são calculados a partir de uma função kernel.

7 As variáveis em $Z$ são semelhantes àquelas em $X$, mas também são adicionadas dummies para o setor de atividade, além de interações e transformações das variáveis para satisfazer o teste de balanceamento, quando necessárias, conforme descrito em Smith e Todd (2005). 


\section{Resultados}

\subsection{Resultados principais}

$\mathrm{Na}$ Tabela 2, são mostrados os resultados estimados para o total de trabalhadores na amostra usando as Equações (1) e (3), separadamente pelo ano de entrada do indivíduo na pesquisa. De acordo com a coluna (1) do painel A, a redução nos rendimentos dos trabalhadores entre 2012 e 2013 foi de $6,1 \%$ para os que passaram por um período de desemprego. Entre 2013 e 2014, a variação estimada nos rendimentos foi de 4,4\%. Já para os indivíduos que estavam empregados em 2014, se tornaram desempregados, e retornaram ao trabalho em 2015, quando a taxa de desemprego já estava muito maior que no ano anterior, a queda estimada nos rendimentos foi de $14,1 \%$. Redução ainda maior foi obtida para o período entre 2015 e 2016, 17,6\%. Entre 2016 e 2017, a queda estimada nos rendimentos como consequência do desemprego foi de $14,5 \%$ em relação àqueles que permaneceram ocupados durante as cinco entrevistas trimestrais da PNAD contínua. ${ }^{8}$

8 Os valores citados no texto referem-se às diferenças estimadas, controlando para diversos fatores, entre o logaritmo do rendimento no grupo de tratamento e de controle, que são reportadas na tabela. Para valores maiores em termos absolutos, como os mostrados nas colunas (3), (4) e (5) do painel A da Tabela 2, por exemplo, essa aproximação não é muito precisa. O coeficiente na coluna (3), - 0,141 corresponde a uma variação de $13,2 \%\left(e^{-0,141}-1=-0,132\right)$. Para $-0,176$, temos $e^{-0,176}-1=-0,161$ e para $-0,145$, temos $e^{-0,145}-1=-0,135$. 
Tabela 2 - Desemprego e variação no logarítimo dos rendimentos do trabalho pelo ano de entrada na PNAD contínua

\begin{tabular}{|c|c|c|c|c|c|}
\hline & (1) & $(2)$ & $(3)$ & (4) & $(5)$ \\
\hline & \multicolumn{5}{|c|}{ Período } \\
\hline & 2012 & 2013 & 2014 & 2015 & 2016 \\
\hline \multicolumn{6}{|l|}{ Painel A } \\
\hline \multirow[t]{2}{*}{ Diferença-em-diferenças } & -0.061 & -0.044 & -0.141 & -0.176 & -0.145 \\
\hline & \multicolumn{5}{|c|}{$[0.017]^{* * *}[0.018]^{* *}[0.017]^{* * *}[0.016]^{* * *}[0.021]^{* * *}$} \\
\hline Observações & 41,422 & 44,119 & 45,359 & 44,698 & 33,204 \\
\hline \multicolumn{6}{|l|}{ Painel B } \\
\hline \multicolumn{6}{|c|}{ Propensity score matching com } \\
\hline \multirow[t]{2}{*}{ diferença-em-diferenças } & -0.032 & -0.002 & -0.077 & -0.111 & -0.078 \\
\hline & {$[0.010]^{* * *}$} & {$[0.010]$} & \multicolumn{3}{|c|}{$[0.010]^{* * *}[0.010]^{* * *}[0.012]^{* * *}$} \\
\hline \multicolumn{6}{|l|}{ Observações } \\
\hline Controle & 68,914 & 74,358 & 76,822 & 74,126 & 55,800 \\
\hline Tratamento & 3,822 & 3,578 & 3,478 & 3,984 & 3,356 \\
\hline \multicolumn{6}{|c|}{$\begin{array}{l}\text { Notas. No painel A, as seguintes variáveis referentes ao período inicial são usadas como } \\
\text { regressores: idade, idade ao quadrado, dummies para escolaridade, gênero, dummies para } \\
\text { região e uma dummy indicando que o indivíduo estava no emprego há pelo menos doze meses. } \\
\text { No painel B, as probabilidades de pertencer ao grupo de tratamento são estimadas usando } \\
\text { model os probit, tendo como variáveis explicativas: idade, idade ao quadrado, dummies para } \\
\text { escolaridade, gênero, dummies para região, para setor de atividade e uma dummy para } \\
\text { indivíduos há } 12 \text { meses ou mais no emprego. } \\
\text { Os erros-padrão são apresentados entre colchetes. } \\
\text { * significativo para o nível de } 10 \%,{ }^{* *} \text { significativo para o nível de } 5 \%, * * * \text { significativo para } \\
\text { o nível de } 1 \%\end{array}$} \\
\hline
\end{tabular}

No painel B, as variações estimadas nos rendimentos como consequência do desemprego são menores em valor absoluto em comparação com as reportadas nas colunas correspondentes do painel A, mas o padrão temporal é semelhante. ${ }^{9}$ Entre 2012 e 2013 , a variação média nos rendimentos dos reempregados é menor em 3,2\% quando comparada à dos que permaneceram empregados, enquanto entre 2013 e 2014 a diferença não chega a ser estatisticamente significativa. De 2012 até 2014, a taxa de desemprego oscilou em torno de $6 \%$. Já a queda nos rendimentos associada ao desemprego para os que entraram na pesquisa em 2014 passa a ser de 7,7\%. Como no painel A, a pior situação é experimentada para a transição entre 2015 , quando a taxa de desemprego chegou a 7,5\%, e 2016, quando passou de $10 \%$. Para esses indivíduos, a redução nos rendimentos foi estimada em 11,1\%. Na análise para o período 2016-2017, quando o desemprego esteve

9 As demais tabelas no artigo se referem apenas aos resultados usando o método de propensity score matching com diferença-em-diferenças. No apêndice, são mostrados os resultados estimados por diferença-em-diferenças correspondentes as demais tabelas dessa subseção. Nota-se que, embora os coeficientes apresentem magnitudes diferentes, as conclusões principais são as mesmas para os dois métodos. 
quase sempre acima de $10 \%$, o ingresso nessa situação teria provocado uma redução de 7,8\% nos rendimentos dos que se reempregaram. ${ }^{10}$

Tabela 3 - Desemprego e variação no logarítimo dos rendimentos do trabalho pelo ano de entrada na PNAD contínua (sem restringir a posição na ocupação de reemprego)

Propensity score matching com diferença-em-diferenças

\begin{tabular}{|c|c|c|c|c|c|}
\hline & (1) & $(2)$ & (3) & (4) & (5) \\
\hline & \multicolumn{5}{|c|}{ Período } \\
\hline & 2012 & 2013 & 2014 & 2015 & 2016 \\
\hline & -0.053 & -0.042 & -0.122 & -0.169 & -0.149 \\
\hline & \multicolumn{5}{|c|}{$[0.010]^{* * *}[0.010]^{* * *}[0.009]^{* * *}[0.010]^{* * *}[0.012]^{* * *}$} \\
\hline \multicolumn{6}{|l|}{ Observações } \\
\hline Controle & 78,140 & 83,694 & 86,252 & 83,196 & 62,022 \\
\hline Tratamento & 4,632 & 4,322 & 4,356 & 5,150 & 4,290 \\
\hline \multicolumn{6}{|c|}{$\begin{array}{l}\text { Notas. As probabilidades de pertencer ao grupo de tratamento são estimadas } \\
\text { usando modelos probit, tendo como variáveis explicativas: idade, idade ao quadrado, } \\
\text { dummies para escolaridade, gênero, dummies para região, para setor de atividade e uma } \\
\text { dummy para indivíduos há } 12 \text { meses ou mais no emprego. } \\
\text { Os erros-padrão são apresentados entre colchetes. } \\
\text { * significativo para o nível de } 10 \%{ }^{* *} \text { significativo para o nível de } 5 \%,{ }^{* * *} \text { significativo para } \\
\text { o nível de } 1 \%\end{array}$} \\
\hline
\end{tabular}

A Tabela 3 mostra os resultados estimados usando o método de propensity score matching com diferença-em-diferenças para uma amostra que não impõe restrição com relação à posição na ocupação dos trabalhadores reempregados na quinta entrevista. De acordo com Reis e Aguas (2014), empregos sem carteira ou por conta própria são um destino importante para indivíduos que desejam sair da condição de desempregados. No caso da Tabela 3, portanto, embora os indivíduos tenham que ser inicialmente empregados com ou sem carteira, podem se reempregar também como trabalhadores por conta própria, funcionários públicos, militares ou empregadores após passarem pelo desemprego. ${ }^{11}$ As reduções nos rendimentos durante os períodos de desemprego mais baixo são estimadas em cerca de $4 \%$ ou $5 \%$. Nos períodos com desemprego mais alto, as reduções nos rendimentos dos reempregados se tornam bem mais acentuadas, oscilando entre $12 \%$ e $17 \%$. Embora o padrão temporal seja semelhante ao mostrado no painel $\mathrm{B}$ da Tabela 2 , as perdas nos rendimentos associadas à passagem pelo desemprego são maiores. Transições para ocupações como conta

\footnotetext{
${ }^{10}$ Para o grupo de controle apenas, são registrados aumentos entre $0,1 \%$ e $4,2 \%$, mas sem um padrão definido entre os diferentes estágios do ciclo econômico.

${ }^{11}$ A mesma condição é adotada para o grupo de controle.
} 
própria, ${ }^{12}$ que representam $90 \%$ dos indivíduos dentro desses novos grupos adicionados na Tabela 3, contribuem para reduções ainda mais acentuadas nos rendimentos de reemprego.

De acordo com os resultados das Tabelas 2 e 3, portanto, transições para o desemprego no período inicial da amostra estão geralmente relacionadas com perdas pequenas nos rendimentos para os que se reempregaram. Apesar da passagem pelo desemprego, esses indivíduos ainda encontraram um mercado de trabalho relativamente favorável, com baixas taxas de desemprego, o que parece ter contribuído para que tenham sido pouco penalizados, ou nem tenham sofrido perdas em alguns casos. O mesmo não acontece nos períodos seguintes. As reduções nos rendimentos do trabalho associadas com saídas para o desemprego entre os indivíduos que se reempregaram em um contexto de desemprego elevado são bem mais acentuadas em relação ao período anterior.

Parte das perdas de rendimentos dos reempregados pode ser atribuída ao tipo de ocupação que os trabalhadores obtêm após passarem pelo desemprego, como já indicado pela comparação entre os resultados das Tabelas 2 e 3. A Figura 1 apresenta as porcentagens de empregados com carteira, entre os integrantes do grupo de tratamento, na primeira e na quinta entrevistas da PNAD contínua. Da amostra de indivíduos ocupados em 2012 que se tornaram desempregados e se reempregaram em 2013, a porcentagem de empregados com carteira, que inicialmente era igual a $61 \%$, passou para 52\% nesse último ano. Note que na Figura 1 o trabalhador necessariamente é um empregado com ou sem carteira na primeira entrevista, mas pode ter qualquer posição na ocupação na quinta entrevista. O resultado para transições entre 2013 e 2014 é muito semelhante ao do período anterior. Com taxas de desemprego mais altas, os empregados com carteira que se tornam desempregados passam a enfrentar maiores dificuldades para se reempregarem também na condição de empregados formais. Para transições entre 2015 e 2016, a porcentagem de empregados com carteira passou de $64 \%$ para $45 \%$. Com isso, as perdas nos rendimentos tendem a ser maiores, pois os rendimentos dos empregados com carteira são, em média, muito maiores que dos trabalhadores por conta própria e empregados sem carteira. Monte et al. (2007) mostram que a remuneração média

12 Entre todos os indivíduos no grupo de tratamento na Tabela 3, 81,4\% se reempregaram como empregados com ou sem carteira, $16,7 \%$ como trabalhadores por conta própria, 1,2\% como funcionários públicos ou militares, e apenas $0,7 \%$ como empregadores. 
dos que se reempregam no setor formal é $70 \%$ superior à média dos que se reempregam no setor informal. ${ }^{13}$

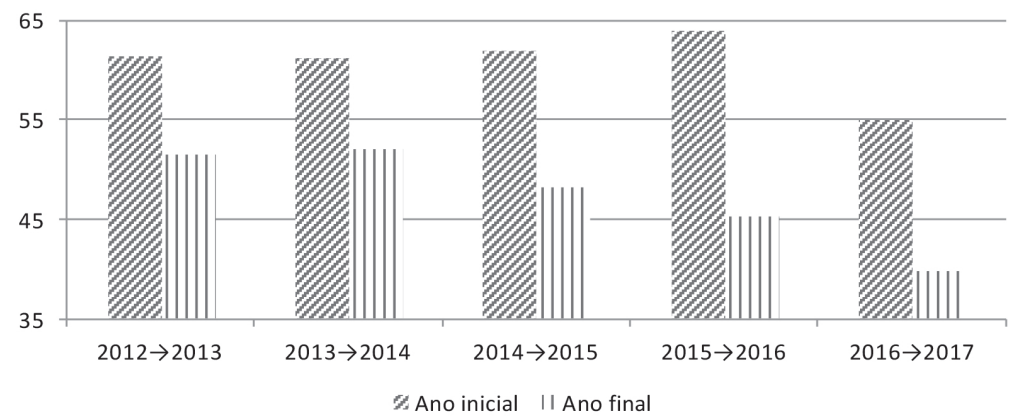

Figura 1 - Porcentagens de com carteira no total de empregados (ano inicial) e no total de reempregados (ano final)

Fonte: PNAD contínua 2012-2017

A amostra inclui trabalhadores com idade entre 21 e 60 anos, empregados na condiçào de com ou sem carteira no período da primeira entrevista da PNAD, e ocupados em qualquer situação na quinta entrevista. São incluídos apenas os indivíduos que passaram pelo desemprego nas entrevistas 2, 3 ou 4.

A análise apresentada na Tabela 4 é restrita, inicialmente, aos empregados com carteira tanto na primeira quanto na quinta entrevista (painel A), e em seguida, aos empregados sem carteira em ambas as entrevistas (painel B). Quando a taxa de desemprego é baixa, os empregados com carteira que após o desemprego conseguem um novo emprego também como empregados formais têm reduções muito pequenas nos rendimentos em relação ao grupo de controle, formado por empregados com carteira que se declararam ocupados em todas as entrevistas. As perdas são maiores nos períodos de desemprego mais elevado, mas não tão grandes quanto as que consideram transições também para empregos sem carteira (na Tabela 2) e para todas as posições na ocupação (na Tabela 3). No apêndice B são mostrados os resultados de estimações semelhantes às apresentadas no painel $\mathrm{B}$ da Tabela 2, mas restringindo a amostra aos inicialmente empregados como com carteira, para que a comparação com a Tabela 4 seja mais adequada.

${ }^{13}$ Para a amostra total usada na Figura 1, a média dos rendimentos dos reempregados como com carteira é $50 \%$ maior que a dos reempregados como conta própria e $70 \%$ superior em comparação com os empregados sem carteira. A média dos rendimentos dos com carteira também é $10 \%$ menor que a dos funcionários públicos e militares e 23\% inferior à dos que se tornaram empregadores, mas as participações desses dois últimos grupos, principalmente o último, são muito pequenas. 
As variações nos rendimentos reportadas na Tabela 4 também são bem menores que as estimativas correspondentes no apêndice B. ${ }^{14}$

Tabela 4 - Desemprego e variação no logarítimo dos rendimentos do trabalho por tipo de transição e ano de entrada na PNAD contínua

\begin{tabular}{|c|c|c|c|c|c|}
\hline & (1) & $(2)$ & (3) & $(4)$ & (5) \\
\hline & \multicolumn{5}{|c|}{ Período } \\
\hline & 2012 & 2013 & 2014 & 2015 & 2016 \\
\hline \multicolumn{6}{|l|}{ Painel A } \\
\hline Empregados com carteira nas & -0.018 & -0.018 & -0.068 & -0.091 & -0.054 \\
\hline entrevistas 1 e 5 & {$[0.009]^{* *}$} & {$[0.009]^{*}$} & {$[0.009]^{* * *}$} & {$[0.009]^{* * *}[$} & {$[0.010]^{* * *}$} \\
\hline \multicolumn{6}{|l|}{ Observações } \\
\hline Controle & 49,610 & 53,588 & 55,130 & 54,492 & 40,736 \\
\hline Tratamento & 1,710 & 1,634 & 1,484 & 1,712 & 1,214 \\
\hline \multicolumn{6}{|l|}{ Painel B } \\
\hline Empregados sem carteira nas & 0.001 & 0.016 & -0.048 & -0.068 & -0.044 \\
\hline entrevistas 1 e 5 & [0.027] & {$[0.026]$} & {$[0.025]^{*}$} & {$[0.026]^{* * *}$} & {$[0.030]$} \\
\hline \multicolumn{6}{|l|}{ Observações } \\
\hline Controle & 11,384 & 13,166 & 13,676 & 12,812 & 9,632 \\
\hline Tratamento & 1,092 & 996 & 1,070 & 1,258 & 1,342 \\
\hline \multicolumn{6}{|c|}{$\begin{array}{l}\text { Notas. As estimativas são obtidas pelo método de propensity escore matching com diferença- } \\
\text { em-diferenças. As probabilidades de pertencer ao grupo de tratamento são estimadas usando } \\
\text { model os probit, tendo como variáveis explicativas: idade, idade ao quadrado, dummies para } \\
\text { escolaridade, gênero, dummies para região, para setor de atividade e uma dummy para } \\
\text { indivíduos há } 12 \text { meses ou mais no emprego. } \\
\text { Os erros-padrão são apresentados entre colchetes. } \\
\text { * significativo para o nível de } 10 \%,{ }^{* *} \text { significativo para o nível de } 5 \%, * * * \text { significativo para } \\
\text { o nível de } 1 \%\end{array}$} \\
\hline
\end{tabular}

Já para as transições entre empregos sem carteira após um determinado período de desemprego, as variações nos rendimentos não são estatisticamente significativas nos dois primeiros períodos. Com taxas de desemprego mais altas, os resultados para 2014 e 2015 no painel B mostram reduções nos rendimentos muito menores que nas Tabelas 2 e 3 , sendo não significativo o resultado para 2016. As variações também são bem menores, em termos absolutos, em comparação com as estimativas no Apêndice $B$, em que a amostra também é limitada aos empregados sem carteira na primeira entrevista.

14 Fatores institucionais, como o salário mínimo, podem contribuir para esse resultado, evitando reduções mais acentuadas nos rendimentos dos trabalhadores com carteira. 
Esses resultados são consistentes com a interpretação de que reduções no salário de reserva dos trabalhadores nos períodos recessivos, em que a demanda por trabalho é menor, parecem desempenhar um papel importante. Com isso, os trabalhadores devem se tornar não apenas mais propensos a aceitar ofertas salariais mais baixas, como também a aceitar empregos no setor informal. Pode-se argumentar também que mesmo que nos períodos mais favoráveis da economia o retorno dos desempregados ao mercado de trabalho seja geralmente mais rápido, não parece muito provável que a perda de capital humano pela duração do desemprego explique os diferenciais acentuados estimados para as variações nos rendimentos, no intervalo de um ano, entre períodos de desemprego baixo e alto. Mesmo assim, a perda de capital humano específico pode ser maior com o mercado de trabalho mais restrito ao aumentar a participação de mudanças de ocupação ou de setor de atividade no processo de reemprego. Já o sinal negativo que o desemprego representaria para os empregadores deveria ser menor nos períodos em que uma proporção maior de indivíduos ingressa nessa situação. ${ }^{15}$

Embora se deva ter cautela com esse tipo de comparação, pelas diferenças entre tipos de dados, métodos e subgrupos analisados, os resultados estimados para o Brasil se situam dentro do intervalo observado para as evidências internacionais apresentadas na seção 2. Em função da enorme heterogeneidade das estimativas, esse fato não chega a ser surpreendente, mas é possível notar também que as perdas estimadas nos rendimentos para o Brasil estão entre as menores, pelo menos em relação às evidências aqui reportadas. Isso pode ser ilustrado, por exemplo, na comparação com os resultados para outro país da América do Sul, o Uruguai, onde a queda nos rendimentos durante os períodos recessivos foi de $34 \%$, e com uma amostra limitada a empregados formais do setor privado. No caso do artigo de Amarante et al. (2014), o período recessivo considerado foi de crise bastante intensa, quando o PIB do país diminui 7,7\% em 2002 depois de ter apresentado queda de 3,8\% no ano anterior. Em 2002, a taxa de desemprego no Uruguai chegou a $17 \%$.

Nota-se também uma diferença bastante acentuada em comparação com os resultados disponibilizados para o Brasil por Menezes-Filho (2003) e Hoeck (2006), que indicam reduções de até 50\%. Apesar de o desemprego ter atingido patamares elevados, as reduções nos rendimentos como consequência da transição do emprego para a condição de desempregado no

${ }^{15}$ Essas interpretações referem-se às comparações entre estágios do ciclo econômico, o que não significa que os rendimentos de reemprego não sejam influenciados por esses fatores. 
Brasil são estimadas aqui em 10\%, ou mesmo em torno de $15 \%$ no caso dos resultados que consideram transições para empregos por conta própria ou das estimativas pelo método de diferença-em-diferenças.

As diferenças de magnitude aqui encontradas em relação a outros estudos para o Brasil chamam a atenção. Em Menezes-Filho (2003), o resultado com queda de $50 \%$ nos rendimentos refere-se aos trabalhadores com longa duração no mesmo emprego, de três anos ou mais. Para esses, a perda de capital humano específico com o reemprego deve ser maior, implicando maiores reduções nos rendimentos. Para trabalhadores com até 3 anos no mesmo emprego a redução foi estimada em 10\%. Já Hoek (2006) considera trabalhadores inicialmente empregados na indústria, que têm rendimentos mais elevados em média. Além disso, o capital humano específico dos trabalhadores da indústria é alto, fazendo com que o potencial para reduções nos rendimentos no processo de reemprego também seja elevado. Apesar de menores que outros resultados na literatura, as reduções nos rendimentos aqui encontradas não podem ser desprezadas, ainda mais que os efeitos podem ser duradouros, dependendo do tempo até que os rendimentos retornem ao nível anterior.

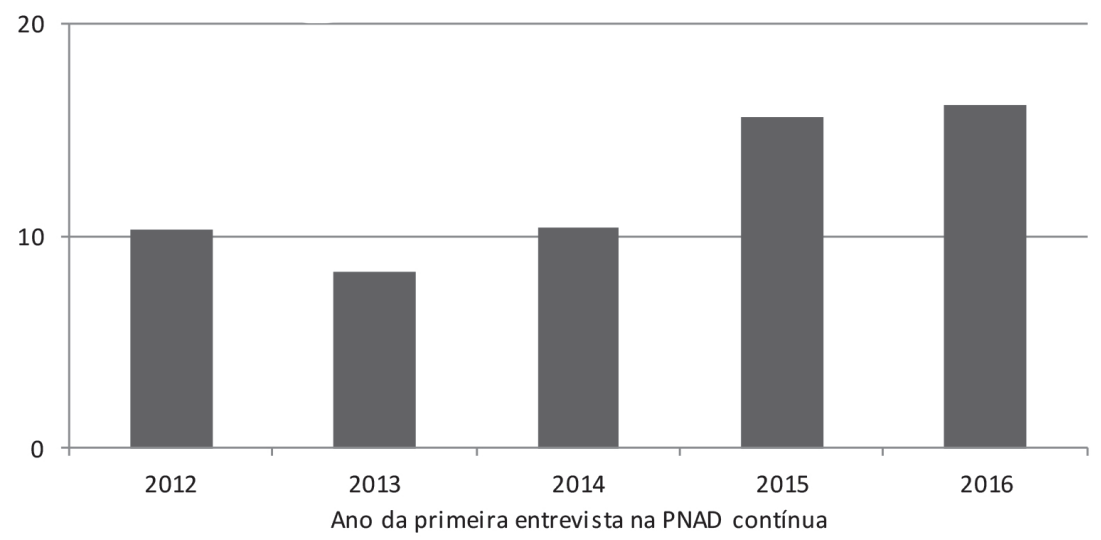

Figura 2 - Porcentagens de indivíduos que depois de terem transitado do emprego para o desemprego não conseguiram se reempregar nas três entrevistas trimestrais restantes

Fonte: PNAD contínua 2012-2017

A amostra inclui trabalhadores com idade entre 21 e 60 anos, empregados na condiçào de com ou sem carteira no período da primeira entrevista da PNAD contínua. São incluídos apenas os indivíduos desempregados na entrevistas 2 . 
As evidências empíricas também mostram que, com a deterioração do mercado de trabalho, os indivíduos que deixam os seus empregos tendem a passar um longo período desempregados. Durante o aumento recente da taxa de desemprego no Brasil, a probabilidade de permanência de um indivíduo no desemprego após um ano é estimada em 63\%, mais de 10 pontos percentuais em relação ao resultado encontrado para um período de desemprego bem mais baixo ocorrido um pouco antes (Reis 2019). A situação dos que transitaram do emprego para o desemprego não se mostra tão ruim, mas também não parece nada confortável. A Figura 2 mostra a situação de indivíduos inicialmente ocupados como empregados com ou sem carteira que transitaram para o desemprego no trimestre seguinte, por ano de entrada na PNAD contínua. Nos anos iniciais, 10\% continuavam desempregados depois de pelo menos 9 meses sem trabalhar. ${ }^{16}$ Em 2015 e 2016, a porcentagem dos que permaneciam desempregados após o mesmo período é superior a $15 \%$. Além da maior dificuldade para se reempregar, os resultados aqui reportados mostram que, com uma conjuntura desfavorável no mercado de trabalho, mesmo para os que conseguem um novo emprego, os rendimentos se tornaram, em média, menores que aqueles que receberiam caso não tivessem ingressado no desemprego.

Alguns estudos recentes têm procurado avaliar quais políticas podem ser adotadas para amenizar os impactos negativos da entrada dos trabalhadores no desemprego. Michaelides et al. (2020) avaliam os efeitos de quatro programas adotados em três diferentes estados norte-americanos, dois na Flórida, um em Idaho e outro em Nevada, para enfrentar as consequências da crise de 2007-09. Todos esses programas ofereciam informações sobre empregos disponíveis, e apenas um dos programas, na Flórida, não incluía monitoramento das atividades de busca por emprego. O programa de Nevada tinha uma característica distinta dos demais ao oferecer um sistema de informações mais detalhado, com aconselhamento profissional para os desempregados. Esse último programa também foi o único a apresentar resultados positivos sobre o emprego e os rendimentos. Os autores concluem que programas baseados apenas no monitoramento dos trabalhadores não são efetivos, e que é fundamental incluir atividades de aconselhamento profissional.

\footnotetext{
${ }^{16}$ Supondo que o indivíduo não teve emprego no intervalo de 3 meses entre duas entrevistas da PNAD contínua.
} 
Os problemas de informação entre os trabalhadores que procuram se reempregar e as firmas oferecendo vagas são analisados por Hellerstein et al. (2019) no contexto da crise de 2007-09 nos Estados Unidos. Esses autores mostram que um mercado de trabalho local com seus agentes bem conectados facilita a processo de reemprego. Esse componente se mostra particularmente importante para os indivíduos com menores rendimentos, que são mais propensos a trabalhar nas proximidades do local de residência.

\subsection{Resultados por subgrupos populacionais}

São apresentadas a seguir as estimativas para alguns subgrupos da população. Enquanto para 2012 as reduções nos rendimentos são ligeiramente maiores para as mulheres, nos períodos de taxa de desemprego mais elevada essa relação se inverte (Tabela 5). Em relação às transições entre 2015 e 2016, por exemplo, as reduções são estimadas em 15\% para os homens e $6 \%$ para as mulheres. Esse resultado pode estar associado às diferentes trajetórias de homens e mulheres após a entrada no desemprego, principalmente em um período recessivo. Parece que enquanto os homens retornam mais rapidamente ao emprego, mesmo que para aceitarem remunerações mais baixas, as mulheres aguardam mais tempo, procurando melhores ofertas de emprego. ${ }^{17}$

${ }^{17}$ Em um contexto mais geral, não restrito ao reemprego, a probabilidade de transição do desemprego para o emprego é muito menor para as mulheres (Menezes-Filho e Picchetti 2000; e Reis 2019). 
Tabela 5 - Desemprego e variação no logarítimo dos rendimentos do trabalho por gênero e pelo ano de entrada na PNAD contínua

\begin{tabular}{|c|c|c|c|c|c|}
\hline & (1) & $(2)$ & (3) & (4) & (5) \\
\hline & \multicolumn{5}{|c|}{ Período } \\
\hline & 2012 & 2013 & 2014 & 2015 & 2016 \\
\hline \multicolumn{6}{|l|}{ Painel A } \\
\hline \multirow[t]{2}{*}{ Mulheres } & -0.043 & 0.003 & -0.065 & -0.060 & -0.056 \\
\hline & {$[0.016]^{* * *}$} & {$[0.015]$} & \multicolumn{3}{|c|}{$[0.015]^{* * *}[0.015]^{* * *}[0.018]^{* *}$} \\
\hline \multicolumn{6}{|l|}{ Observações } \\
\hline Controle & 27,670 & 31,006 & 32,370 & 32,088 & 23,936 \\
\hline Tratamento & 1,616 & 1,534 & 1,478 & 1,728 & 1,446 \\
\hline \multicolumn{6}{|l|}{ Painel B } \\
\hline \multirow[t]{2}{*}{ Homens } & -0.025 & -0.005 & -0.082 & -0.150 & -0.096 \\
\hline & {$[0.012]^{* *}$} & [0.013] & \multicolumn{3}{|c|}{$[0.012]^{* * *}[0.013]^{* * *}[0.016]^{* * *}$} \\
\hline \multicolumn{6}{|l|}{ Observações } \\
\hline Controle & 40,806 & 43,222 & 43,906 & 42,054 & 31,464 \\
\hline Tratamento & 2,204 & 2,046 & 2,002 & 2,254 & 1,912 \\
\hline \multicolumn{6}{|c|}{$\begin{array}{l}\text { Notas. As estimativas são obtidas pelo método de propensity escore matching com diferença- } \\
\text { em-diferenças. As probabilidades de pertencer ao grupo de tratamento são estimadas usando } \\
\text { model os probit, tendo como variáveis explicativas: idade, idade ao quadrado, dummies para } \\
\text { escolaridade, dummies para região, para setor de atividade e uma dummy para indivíduos } \\
\text { há } 12 \text { meses ou mais no emprego. }\end{array}$} \\
\hline \multicolumn{6}{|c|}{$\begin{array}{l}\text { Os erros-padrão são apresentados entre colchetes. } \\
\text { * significativo para o nivel de } 10 \%,{ }^{* *} \text { significativo para o nível de } 5 \%,{ }^{* *} \text { significativo para } \\
\text { o nível de } 1 \%\end{array}$} \\
\hline
\end{tabular}

Embora Couch e Placzek (2010) argumentem que os empregadores teriam menos incentivos para investirem no capital humano das mulheres, por essas apresentarem menor duração média no emprego que os homens, o que implicaria perdas menores de capital humano para as mulheres, os resultados por gênero são semelhantes para uma série de estudos reportados por esses autores. Já Manzoni e Mooi-Reci (2020) argumentam que, como entre as mulheres as saídas do mercado de trabalho são mais frequentes, o desemprego não é interpretado pelos empregadores como um sinal tão negativo da produtividade quanto para os homens.

Como os trabalhadores mais escolarizados devem possuir níveis mais elevados de capital humano específico que os menos escolarizados, espera-se que as maiores reduções nos rendimentos como consequência do ingresso no desemprego sejam observadas entre os mais escolarizados. MenezesFilho (2003) encontra evidências consistentes com esse argumento para o Brasil. Os resultados por grupo de escolaridade na Tabela 6, porém, são 
muito semelhantes entre indivíduos com pelo menos o ensino médio completo e aqueles menos escolarizados.

Tabela 6 - Desemprego e variação no logarítimo dos rendimentos do trabalho por escolaridade e pelo ano de entrada na PNAD contínua

\begin{tabular}{|c|c|c|c|c|c|}
\hline & $(1)$ & $(2)$ & $(3)$ & (4) & (5) \\
\hline & \multicolumn{5}{|c|}{ Período } \\
\hline & 2012 & 2013 & 2014 & 2015 & 2016 \\
\hline \multicolumn{6}{|l|}{ Painel A } \\
\hline Escolaridade: até o ensino & -0.045 & -0.003 & -0.086 & -0.127 & -0.076 \\
\hline médio incompleto & {$[0.014]^{* * *}$} & {$[0.014]$} & {$[0.014]^{* * *}$} & {$[0.015]^{* * *}$} & {$[0.019]^{* * *}$} \\
\hline \multicolumn{6}{|l|}{ Observações } \\
\hline Controle & 31,738 & 33,620 & 33,645 & 32,224 & 22,188 \\
\hline Tratamento & 1,886 & 1,738 & 1,711 & 1,952 & 1,520 \\
\hline \multicolumn{6}{|l|}{ Painel B } \\
\hline Pelo menos o ensino & -0.047 & -0.025 & -0.084 & -0.116 & -0.075 \\
\hline médio completo & {$[0.013]^{* * *}$} & {$[0.014]^{*}$} & {$[0.013]^{* * *}$} & {$[0.013]^{* * *}$} & {$[0.015]^{* * *}$} \\
\hline \multicolumn{6}{|l|}{ Observações } \\
\hline Controle & 34,177 & 38,059 & 40,420 & 39,661 & 30,044 \\
\hline Tratamento & 1,783 & 1,689 & 1,654 & 1,877 & 1,588 \\
\hline \multicolumn{6}{|c|}{$\begin{array}{l}\text { Notas. As estimativas são obtidas pelo método de propensity escore matching com diferença- } \\
\text { em-diferenças. As probabilidades de pertencer ao grupo de tratamento são estimadas usando } \\
\text { model os probit, tendo como variáveis explicativas: idade, idade ao quadrado, dummies para } \\
\text { escolaridade, gênero, dummies para região, para setor de atividade e uma dummy para } \\
\text { indivíduos há } 12 \text { meses ou mais no emprego. } \\
\text { Os erros-padrão são apresentados entre colchetes. } \\
\text { * significativo para o nível de } 10 \% \text {, }{ }^{* *} \text { significativo para o nível de } 5 \%, * * * \text { significativo para } \\
\text { o nível de } 1 \%\end{array}$} \\
\hline
\end{tabular}

Trabalhadores mais velhos, normalmente com mais capital humano específico acumulado através da experiência no emprego, devem sofrer reduções maiores nos rendimentos de reemprego do que os trabalhadores mais novos. Os estudos reportados por Couch e Placzek (2010) para os Estados Unidos apresentam resultados consistentes com esse argumento. Amarante et al. (2014), no entanto, não encontram diferenças entre grupos etários para o Uruguai. Os resultados na Tabela 7 são semelhantes ao desse último estudo. Embora as reduções nos rendimentos para os mais velhos sejam maiores, as diferenças em relação aos mais jovens são muito pequenas. Nota-se também que para todos os subgrupos analisados nessa subseção as reduções nos rendimentos de reemprego são maiores nos períodos de desemprego mais alto. 
Tabela 7 - Desemprego e variação no logarítimo dos rendimentos do trabalho por faixa etária e pelo ano de entrada na PNAD contínua

\begin{tabular}{|c|c|c|c|c|c|}
\hline & (1) & $(2)$ & (3) & (4) & (5) \\
\hline & \multicolumn{5}{|c|}{ Período } \\
\hline & 2012 & 2013 & 2014 & 2015 & 2016 \\
\hline \multicolumn{6}{|l|}{ Painel A } \\
\hline \multirow[t]{2}{*}{ Idade: 40 anos ou menos } & -0.032 & -0.013 & -0.073 & -0.114 & -0.079 \\
\hline & {$[0.012]^{* * *}$} & {$[0.012]$} & \multicolumn{3}{|c|}{$[0.012]^{* * *}[0.012]^{* * *}[0.015]^{* * *}$} \\
\hline \multicolumn{6}{|l|}{ Observações } \\
\hline Controle & 42,582 & 46,006 & 46,260 & 44,216 & 32,604 \\
\hline Tratamento & 2,872 & 2,682 & 2,534 & 2,818 & 2,282 \\
\hline Painel B & -0.044 & 0.037 & -0.071 & -0.110 & -0.081 \\
\hline Idade: 41 anos ou mais & \multicolumn{5}{|c|}{$[0.018]^{* *}[0.019]^{* *}[0.017]^{* * *}[0.018]^{* * *}[0.021]^{* * *}$} \\
\hline \multicolumn{6}{|l|}{ Observações } \\
\hline Controle & 24,266 & 25,822 & 27,954 & 28,162 & 21,210 \\
\hline Tratamento & 848 & 818 & 840 & 1,042 & 956 \\
\hline \multicolumn{6}{|c|}{$\begin{array}{l}\text { Notas. As esti mativas são obtidas pelo métodp de propensity escore matching com diferença- } \\
\text { em-diferenças. As probabilidades de pertencer ao grupo de tratamento são estimadas usando } \\
\text { model os probit, tendo como variáveis explicativas: idade, idade ao quadrado, dummies para } \\
\text { escolaridade, gênero, dummies para região, para setor de atividade e uma dummy para } \\
\text { indivíduos há } 12 \text { menos ou mais no emprego. } \\
\text { As estatísticas-t são apresentados entre colchetes. } \\
\text { * significativo para o nível de } 10 \%,{ }^{* *} \text { significativo para o nível de } 5 \%,{ }^{* * *} \text { significativo para } \\
\text { o nível de } 1 \%\end{array}$} \\
\hline
\end{tabular}

\section{Conclusões}

De acordo com os resultados estimados neste artigo, durante períodos em que a conjuntura do mercado de trabalho é mais favorável, as saídas para o desemprego apresentam efeitos sobre os rendimentos de reemprego pequenos, que em muitos casos não são estatisticamente diferentes de zero. Nos períodos caracterizados por piores condições do mercado de trabalho, porém, o efeito do desemprego passa a representar uma diminuição entre $10 \%$ e 15\% nos rendimentos dos indivíduos que posteriormente conseguem se reempregar. Mesmo essas estimativas para um período de desemprego elevado são bem inferiores às perdas nos rendimentos obtidas em outros estudos para o Brasil, em que as amostras se restringiam a trabalhadores da indústria ou do setor formal com muito tempo no mesmo emprego. 
O mesmo comportamento para as variações nos rendimentos como consequência do ingresso no desemprego ao longo dos ciclos econômicos também é observado em diversos outros países. Nota-se também que os resultados aqui estimados para o Brasil indicam reduções nos rendimentos do trabalho relativamente baixas para o padrão internacional, embora nada desprezíveis.

Uma parte dessas perdas nos rendimentos se mostra associada a transições de empregos com carteira para empregos no setor informal. Os empregos desse último tipo são geralmente de pior qualidade e oferecem remunerações mais baixas do que no setor formal, mas se tornam uma alternativa atraente para os que ingressam no desemprego, principalmente nos períodos em que a situação do mercado de trabalho é desfavorável, pela maior facilidade para se reempregar. As reduções nos rendimentos nesse caso, no entanto, costumam ser acentuadas.

Nos períodos de desemprego elevado, principalmente, o fluxo de trabalhadores que se deslocam do emprego para o desemprego parece envolver custos que não se limitam ao efeito mais direto, representado pela perda dos rendimentos do trabalho para esses indivíduos. Além de muitos enfrentarem um processo demorado de retorno ao emprego, os resultados aqui apresentados indicam que, mesmo após se reempregarem, os trabalhadores experimentam, em média, uma redução nos rendimentos em relação ao que receberiam caso não tivessem entrado no desemprego. Dessa forma, espera-se que a trajetória de recuperação do poder de compra seja geralmente lenta, influenciando possivelmente não apenas o bem-estar do próprio indivíduo, mas também de outros integrantes do domicílio. 


\section{Referências}

Amarante, Verónica, Rodrigo Arim and Andrés Dean. 2014. "The Effects of Being Out of the Labor Market on Subsequent Wages: Evidence for Uruguay”. Journal of Labor Research 35, pp.39-62.

Burda, Michael and Antje Mertens. 2001. "Estimating wage losses of displaced workers in

Germany". Labour Economics 8, no. 1, pp. 15-41.

Couch, Kenneth and Dana W. Placzek. 2010. "Earnings Losses of Displaced Workers Revisited". The American Economic Review 100, no. 1, pp. 572-589

Davis, Steven and Till M. von Wachter. 2011. "Recessions and the cost of job loss". NBER Working Paper 17638.

Farber, Henry. 1993. "The Incidence and Costs of Job Loss: 1982-91.” Brookings Papers on Economic Activity: Microeconomics 1: 73-132.

Farber, Henry. 2015. "Job loss in the great recession and its aftermath: US evidence from the Displaced Workers". NBER Working Paper 21216

Farber, Henry. 2017. "Employment, hours, and earnings consequences of job loss: US evidence from the Displaced Workers Survey. Journal of Labor Economics 35, no. S1.

Gregory, M. and R. Jukes. 2001. "Unemployment and subsequent earnings: Estimating scarring among British men 1984-94.” The Economic Journal 111(475): 607-625.

Heckman, J., H. Ichimura, and P. Todd. 1997. "Matching as an econometric evaluation estimator: evidence from evaluating a job training program". Review of Economic Studies 64, no. 4, pp. 605-654.

Hellerstein, J.; M. Kutzbach and D. Neumark. 2019. "Labor market networks and recovery from mass layoffs: evidence from the great recession period.” NBER Working Paper 21262.

Hijzen A, R. Upward, P,Wright. 2010. "The income losses of displaced workers." Journal of Human Resources 45, no.1:243-269.

Hoek, Jasper. 2006. "Life Cycle Effects of Job Displacement in Brazil”. IZA DP No. 2291.

Jacobson, Louis S., Robert J. LaLonde, and Daniel G. Sullivan. 1993. "Earnings Losses of Displaced Workers”. The American Economic Review 83: 685-709.

Kletzer, L. 1998. “Job displacement”. Journal of Economic Perspectives 12, no.1:115-136.

Korkeamäki, O. and T. Kyyrä. 2014. "A distributional analysis of earnings losses of displaced workers in an economic depression and recovery”. Oxford Bulletin of Economics and Statistics 76, no.4, 565-88.

Manzoni, A.; I. Mooi-Reci. 2020. "The cumulative disadvantage of unemployment: Longitudinal evidence across gender and age at first unemployment in Germany". PLOS ONE 15, no.6: e0234786.

Menezes-Filho, N.; P. Picchetti. 2000. "Determinantes do desemprego no Brasil. " Pesquisa e Planejamento Econômico 30, no. 1, abr.

Menezes-Filho, N. 2003. "The Costs of Displacement in Brazil”. Mimeo.

Michaelides, M.; P. Mueser and J. Smith. 2020. "Do Reemployment Programs for the Unemployed Work for Youth? Evidence from the Great Recession in the United States." IZA DP No. 13324

Monte, P.; T. Araújo e R. Lima. 2007. "Primeiro emprego e reemprego: análise de inserção ocupacional e duração do desemprego no Brasil metropolitano". Economia e Desenvolvimento 7, no. 1, p. 139-177.

Reis, M. e M. Aguas. 2014. Duração do desemprego e transições para o emprego formal, a inatividade e a informalidade. Economia Aplicada 18 9(1).

Reis, M. 2019. "Como as condições do mercado de trabalho influenciam as transições do desemprego para o emprego?". Texto para discussão do IPEA, no. 2488.

Rosenbaum, P., and D. Rubin. 1983. The central role of the propensity score in observational studies for causal effects. Biometrika 70, 41-55. 
Ruhm, Christopher. 1991. “Are Workers Permanently Scarred by Job Displacements?”

American Economic Review 81, no.1, 319-323.

Schmieder, J.; T. von Wachter e J. Heining. 2020. "The Costs of Job Displacement over the Business Cycle and Its Sources: Evidence from Germany". Mimeo.

Smith, J., and P. Todd. 2005. Does matching overcome Lalonde's critique of nonexperimental estimators? Journal of Econometrics 125, no. 1-2, 305-53.

Stevens, Ann Huff. 1997. "Persistent Effects of Job Displacement: The Importance of Multiple Job Losses." Journal of Labor Economics 15, no.1: 165-88.

Topel, Robert. 1990. "Specific Capital and Unemployment: Measuring the Costs and Consequences of Job Loss." Carnegie-Rochester Conference Series on Public Policy 33: 181-214.

Verho, J. 2020. "Economic Crises and Unemployment Persistence: Analysis of Job Losses During the Finnish

Recession of the 1990s". Economica 87, p. 190-216

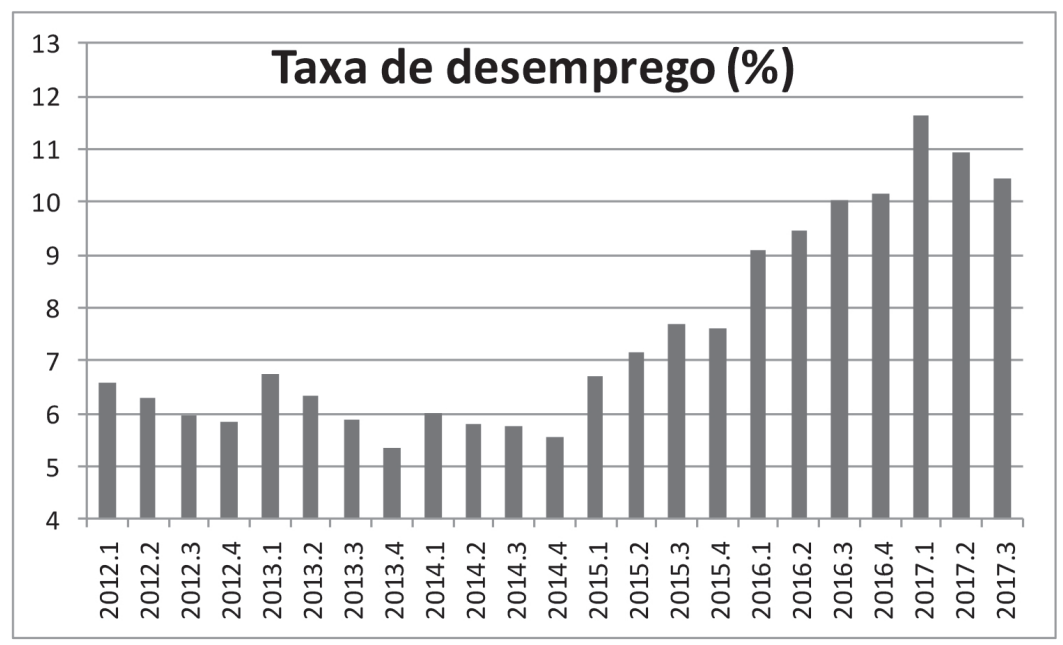

Figura 1A 
Tabela B.1 - Desemprego e variação no logarítimo dos rendimentos do trabalho pela posição inicial do trabalhador e ano de entrada na PNAD contínua

\begin{tabular}{|c|c|c|c|c|c|}
\hline & $(1)$ & $(2)$ & $(3)$ & (4) & $(5)$ \\
\hline & \multicolumn{5}{|c|}{ Período } \\
\hline & 2012 & 2013 & 2014 & 2015 & 2016 \\
\hline \multicolumn{6}{|l|}{ Painel A } \\
\hline Empregados com carteira & -0.098 & -0.07 & -0.144 & -0.173 & -0.153 \\
\hline & \multicolumn{5}{|c|}{$[0.009]^{* * *}[0.009]^{* * *}[0.009]^{* * *}[0.009]^{* * *}[0.010]^{* * *}$} \\
\hline \multicolumn{6}{|l|}{ Observações } \\
\hline Controle & 52,866 & 56,580 & 57,882 & 57,786 & 42,836 \\
\hline Tratamento & 2,258 & 2,128 & 2,014 & 2,340 & 1,714 \\
\hline \multicolumn{6}{|l|}{ Painel B } \\
\hline \multirow{2}{*}{ Empregados sem carteira } & 0.031 & 0.064 & -0.002 & -0.030 & -0.023 \\
\hline & {$[0.022]$} & {$[0.021]^{* * *}$} & {$[0.021]$} & {$[0.022]$} & {$[0.026]$} \\
\hline \multicolumn{6}{|l|}{ Observações } \\
\hline Controle & 15,988 & 17,928 & 18,126 & 16,748 & 12,464 \\
\hline Tratamento & 1,562 & 1,450 & 1,464 & 1,640 & 1,640 \\
\hline \multicolumn{6}{|c|}{$\begin{array}{l}\text { Notas. As estimativas são obtidas pelo método de propensity escore matching com diferença- } \\
\text { em-diferenças. As probabilidades de pertencer ao grupo de tratamento são estimadas usando } \\
\text { model os probit, tendo como variáveis explicativas: idade, idade ao quadrado, dummies para } \\
\text { escolaridade, gênero, dummies para região, para setor de atividade e uma dummy para } \\
\text { indivíduos há } 12 \text { meses ou mais no emprego. } \\
\text { Os erros-padrão são apresentados entre colchetes. } \\
\text { * significativo para o nível de } 10 \% \text {, ** significativo para o nível de } 5 \% \text {, *** significativo para } \\
\text { o nível de } 1 \%\end{array}$} \\
\hline
\end{tabular}

Tabela C.1- Desemprego e variação no logarítimo dos rendimentos do trabalho pelo ano de entrada na PNAD contínua (sem restringir a posição na ocupação de reemprego) Diferença-em-Diferenças

\begin{tabular}{lccccc}
\hline & $(1)$ & $(2)$ & $(3)$ & $(4)$ & $(5)$ \\
\hline & \multicolumn{5}{c}{ Período } \\
& 2012 & 2013 & 2014 & 2015 & 2016 \\
\cline { 2 - 6 } & \multicolumn{7}{c}{0.141} & -0.176 & -0.145 \\
Coeficiente & -0.061 & -0.045 & -0.141 & \\
Erro-padrão & $0.017]^{* * *}$ & $0.018]^{* *}$ & {$[0.017]^{* * *}[0.016]^{* * *}[0.021]^{* * *}$} \\
\hline Observações & 41,407 & 44,114 & 45,355 & 44,694 & 33,203 \\
\hline
\end{tabular}

Notas. As seguintes variáveis referentes ao período inicial são usadas como regressores:

idade, idade ao quadrado, dummies para escolaridade, gênero, dummies para região e uma dummy indicando que o indivíduo estava no emprego há pelo menos doze meses. Os erros-padrão são apresentados entre colchetes.

* significativo para o nível de $10 \%,{ }^{* *}$ significativo para o nível de $5 \%,{ }^{* * *}$ significativo para o nível de $1 \%$ 
Tabela C.2 - Desemprego e variação no logarítimo dos rendimentos do trabalho pelo ano de transição e ano de entrada na PNAD contínua Diferença-em-Diferenças

\begin{tabular}{|c|c|c|c|c|c|}
\hline & (1) & (2) & (3) & (4) & (5) \\
\hline & \multicolumn{5}{|c|}{ Período } \\
\hline & 2012 & 2013 & 2014 & 2015 & 2016 \\
\hline \multicolumn{6}{|l|}{ Painel A } \\
\hline Empregados com carteira nas & -0.046 & -0.042 & -0.082 & -0.113 & -0.061 \\
\hline entrevistas 1 e 5 & {$[0.018]^{* *}$} & {$[0.022]^{*}$} & {$[0.022] * * *$} & {$[0.022]^{* * *}[$} & {$[0.020]^{* * *}$} \\
\hline Observações & 25,701 & 27,662 & 28,580 & 28,315 & 21,184 \\
\hline \multicolumn{6}{|l|}{ Painel B } \\
\hline Empregados sem carteira nas & 0.019 & 0.061 & -0.121 & -0.084 & -0.027 \\
\hline entrevistas 1 e 5 & {$[0.045]$} & [0.040] & {$[0.038]^{* * *}$} & {$[0.037]^{* *}$} & [0.039] \\
\hline Observações & 6,264 & 7,104 & 7,389 & 7,064 & 5,509 \\
\hline
\end{tabular}

Notas. As seguintes variáveis referentes ao período inicial são usadas como regressores: idade, idade a o quadrado, dummies para escolaridade, gênero, dummies para região e uma dummy indicando que o indivíduo estava no emprego há pelo menos doze meses. Os erros-padrão são apresentados entre colchetes.

Os erros-padrão são apresentados entre colchetes.

* significativo para o nível de $10 \%$, ** significativo para o nível de $5 \%$, *** significativo para o nível de $1 \%$ 